\title{
Biophysics of Pole Formation of Gram-positive Rods
}

\author{
By A. L. KOCH ${ }^{1 *}$ AND I. D. J. BURDETT ${ }^{2}$ \\ ${ }^{1}$ Department of Biology, Indiana University, Bloomington, Indiana 47405, USA \\ ${ }^{2}$ National Institute for Medical Research, The Ridgeway, Mill Hill, London NW7 IAA, UK
}

(Received 13 January 1986; revised 20 June 1986)

During pole formation in Bacillus subtilis the inner and outer surfaces of the nascent pole are enlarged by almost exactly the same extent. This means that the stress is almost uniformly distributed throughout the polar wall. This differs from the situation in the cylindrical side wall, where most of the stress is exerted in the outer portions of the intact wall. Because the stress is shared more uniformly, the maximum strain in any part of the polar wall is reduced, compared with the maximum strain within the side wall. The lowered stress may account, in part, for the resistance of the polar wall to hydrolysis by autolytic enzymes under certain conditions. The shape of the newly completed pole is significantly different from the spherical shape that the hydrostatic pressure would tend to produce. It does, however, achieve the shape that maximizes the polar volume under the restrictions arising due to expansion along the circumference not being possible near the junction of cylindrical and polar wall.

\section{INTRODUCTION}

A Gram-positive rod such as Bacillus subtilis divides by forming a cross-wall by centripetal addition of murein. From the median sections assembled by Burdett \& Higgins (1978) it is reasonable to suppose that most of the peptidoglycan is added and polymerized to the innermost layer of the developing septum and that little or none is added to the lateral faces. After a delay, the cross-wall is split from the outside, also centripetally. Even though the surface area increases by about $50 \%$, very little new wall appears to be added while the flat septum is converted to the rounded pole. This could have been anticipated from the knowledge that the nascent muropeptides as well as the transpeptidase and transglycosylase are bound to the cytoplasmic membrane (see Koch et al., 1985). This militates against murein being added throughout the thickness of the wall while the poles are formed and suggests that the increase in the surface area with little change in thickness results in a decrease in density of the polar murein. The preceding paper (Koch \& Burdett, 1986) also supports this conclusion, providing independent confirmation of the 'split-and-stretch' model, which was originally based on considerations of the changes in shape of the nascent pole as it develops (Koch, 1983; Burdett \& Koch, 1984).

The present paper provides further comparison of the predictions of this model with published experiments. This comparison leads to a significant modification of a detail of the model.

\section{THE STABILITY OF THE GRAM-POSITIVE POLE}

The shape of the mature pole can change little from the time it is first formed. This conclusion follows from the metabolic stability of the pole. After a lag, turnover of the side wall material of Gram-positive rods results in the rapid release of murein components into the medium (for review see Koch \& Doyle, 1985). This phenomenon is due to the obligate movement of the cylindrical wall material from inside to outside as elongation takes place (see Koch et al., 1981). In contrast, the poles of Gram-positive rods (and cocci) undergo turnover very much more slowly (Archibald \& Coapes, 1976; Doyle et al., 1983; Mobley et al., 1984). In long term 
experiments designed to measure the turnover of this compartment of labelled wall material, it was found that pole label was released at about $5 \%$ per doubling time under the usual growth conditions (see Koch \& Doyle, 1985; G. Kirchner, R. J. Doyle \& A. L. Koch, unpublished). The rate was not decreased even when amidases and glycosaminidases, liberated from the poles or cylindrical portions of the same or other cells, were prevented from attaching to polar wall when an excess of purified wall was added to the medium.

\section{THE SHAPE OF THE B. SUBTILIS POLE}

The biophysical forces acting to determine the morphology of the pole can in part be deduced from the mathematical shape of the pole. In earlier work (Burdett \& Higgins, 1978; Koch, 1983) the development of the pole was studied by analysing median section electron micrographs of cells chosen at random from a balanced, growing culture. Further refinement has recently been obtained by a more detailed analysis of selected cells in defined phases of pole formation (Burdett \& Koch, 1984). In this latter work, we studied three groups of cells: ( $i$ ) cells that had just initiated septum splitting; (ii) cells that were just about to complete the process; and (iii) cells that had just completed pole formation. During the initial splitting of the cross-wall the nascent poles formed a larger angle with the extension of the side wall than the angle observed in later stages of pole formation (see Fig. 6 of Burdett \& Higgins, 1978). Measurements of diameters of curvature and linear dimensions of poles (Burdett \& Koch, 1984) showed that the completed pole was blunter than a hemisphere and did not fit a number of explicit mathematical models proposed in the earlier literature (see Burdett \& Koch, 1984) including: (i) the parabola; (ii) the prolate ellipse; (iii) the Cassinian; and (iv) the zonal dome.

\section{VARIATION OF STRAIN THROUGHOUT THE POLAR WALL}

The side wall grows and elongates by an inside-to-outside growth mechanism, as first convincingly demonstrated by Pooley (1976). It was pointed out by Koch et al. (1981) that because peptidoglycan is added diffusely in a non-extended conformation to the inner surface it can expand later as it moves peripherally to assume the stress due to hydrostatic pressure. We suggested that this is the basis of the mechanism that Gram-positive rods use to elongate. Older portions of the side wall rupture and are usually subsequently discarded, although they may remain attached to the cell as fibrous or capsular material. For biochemical reasons the stress must be zero at the sites where cross-linking takes place, whereas it reaches a maximum in outer layers of the wall just before rupture takes place. Recently, we have presented calculations which show that the wall stretches about two-fold as it is displaced outward from the cell membrane to the outermost intact layer (Koch \& Doyle, 1985).

The polar wall would be expected to show a very different pattern of stretch. Unsplit septal wall would experience little stress and should therefore exhibit little strain. As the septum splits to form two poles of equal thickness (Koch et al., 1985), stress and strain would appear within the nascent pole and in the unsplit portion of the septum. The following question can be asked: given that no additional material is added during the splitting phase, what is the distribution of stresses throughout the thickness of the polar wall? The degree of expansion on both faces can be estimated directly from polar surface area measurements of the median sections of $B$. subtilis. The mean outside surface area of 10 nearly divided cells was $0.479 \mu \mathrm{m}^{2}$ and that of the inside surface was $0.392 \mu \mathrm{m}^{2}$. The ratio, 1.22 , was almost identical to that of completed poles of the same cells: $0.522 / 0.433 \mu \mathrm{m}^{2}=1.21$. It can be seen that expansion of area is nearly the same on both faces, and consequently the stress is nearly uniform throughout the wall.

In a more general way, this can also be shown for two approximate shapes: spherical and planar (Bacillus anthracis-like) (Table 1). Consequently, the strain, and therefore the stress, is distributed quite uniformly throughout the thickness no matter what exact shape the pole possesses. This implies that none of the polar wall is under as strong a local tension as the most stressed outer side wall, but that all of the pole is under more strain than the nascent layer in the side wall. This more uniform distribution of stress, and therefore, the lower maximal stress, may 
Table 1. Relative expansion of the inside and outside surface of the pole

For both pole shape models, the ratio of the expansion of the outside face to that of the inner face is computed. Subscripts o and i stand for outside and inside. $r$ is the maximum radius and $z$ or $x$ is the maximum axial height.

\begin{tabular}{|c|c|c|}
\hline & Geometric & el of pole \\
\hline & Spherical segment & Bacillus anthracis-like* \\
\hline Pole surface area & $\pi\left(r^{2}+z^{2}\right)$ & $\pi\left(r^{2}+2 r x\right)$ \\
\hline Septum area & $\pi r^{2}$ & $\pi r^{2}$ \\
\hline Expansion ratio & $1+z^{2} / r^{2}$ & $1+2 x / r$ \\
\hline & 1.52 & 1.52 \\
\hline$r_{0}$ & $0.2975 \mu \mathrm{m}$ & $0.2975 \mu \mathrm{m}$ \\
\hline$z_{0}, x_{0}$ & $0.2975 \times(0.52)^{0.5}=0.2145 \mu \mathrm{m}$ & $0.2975 \times 0.52 / 2=0.0774 \mu \mathrm{m}$ \\
\hline$r_{i}$ & $0.2145-0.024 t=0.1905 \mu \mathrm{m}$ & $0.0774-0.024 \ddagger=0.0534 \mu \mathrm{m}$ \\
\hline$z_{\mathrm{i}}, x_{\mathrm{i}}$ & $0.2145-0.024 \ddagger=0.1905 \mu \mathrm{m}$ & $0.0774-0.024 \ddagger=0.0534 \mu \mathrm{m}$ \\
\hline & 1.4102 & $1 \cdot 3901$ \\
\hline$E_{0} / E_{\mathrm{i}}$ & 1.08 & 1.09 \\
\hline & $\begin{array}{l}\text { pole plus cylindrical 'pill box' } \\
\text { of } 1.52 \text { taken as the mean of } \\
\text { ickness of the wall is taken to }\end{array}$ & $\begin{array}{l}\text { lable values of } E \text {. } \\
4 \mu \mathrm{m} \text {. }\end{array}$ \\
\hline
\end{tabular}

be part of the explanation of the relative resistance of poles to autolysis, and their greatly reduced rate of pole turnover.

There is also a physical factor involved which arises because of the difference in the energetics between spherical and cylindrical surfaces. $P=2 T / r$ and $P=T / r$ describe the equilibrium situation for the two cases respectively. In these equations $P$ is the pressure, $T$ is the analogue of surface tension and $r$ is the radius. Therefore, for the same radius and pressure, the surface tension in the spherical portion is half that of the cylindrical wall. A third conjecture to explain the difference in resistance to autolysis of the polar wall and the cylindrical wall is that autolysins are preferentially localized in the cylindrical wall.

\section{EXPANSION OF THE CROSS-WALL DURING POLE FORMATION}

Because the cross-wall is not normally completed before splitting commences, there is the possibility that wall laid down on the inside of the centripetally growing annulus may be stretched to some degree before additional wall is added inside it (this is the case in Streptococcus faecium; Higgins \& Shockman, 1976; A. L. Koch \& M. L. Higgins, unpublished). In that case, more peptidoglycan units would be used in the construction of the pole than if enlargement of the septal ring did not take place. In our previous analysis we assumed this to be the case for $B$. subtilis (Burdett \& Koch, 1984), but we now reject this possibility. An assessment of this contingency is possible by the re-analysis of the data given by Burdett $\&$ Higgins (1978). They measured nascent poles in various stages of development, taking the area of the nascent pole, $P_{\mathrm{a}}$, as an index of development of the pole. We estimated $P_{\mathrm{af}}$, the final value of $P_{\mathrm{a}}$, by extrapolating the plot of $D_{\min }$, the minimum diameter of the unsplit septum, versus $P_{\mathrm{a}}$ to the x-axis, where $D_{\min }=0 . P_{\text {af }}$ was $0.39 \mu \mathrm{m}^{2}$. Then at any stage of pole development, the pole area yet to be formed, designated $\left(P_{\mathrm{af}}-P_{\mathrm{a}}\right)$, will be created by stretching the planar septal wall. As before (Burdett \& Koch, 1984) the expansion factor, $E$, is defined as the area of polar wall after splitting relative to the area of septal wall before splitting. $E$, throughout pole formation, can be calculated by dividing $P_{\mathrm{af}}-P_{\mathrm{a}}$ by the area of the remaining unsplit septum, $\pi D_{\min }{ }^{2} / 4$ :

$$
E=4\left(P_{\mathrm{af}}-P_{\mathrm{a}}\right) / \pi D_{\min }^{2}
$$

Values of $E$ versus $P_{\text {a }}$ calculated from the data of Burdett \& Higgins (1978) are shown in Fig. 1. The values show some variation, and the variation is particularly large near the completion of pole development when the computation is not accurate. The mean ( \pm SD) of all the values is 1.67 


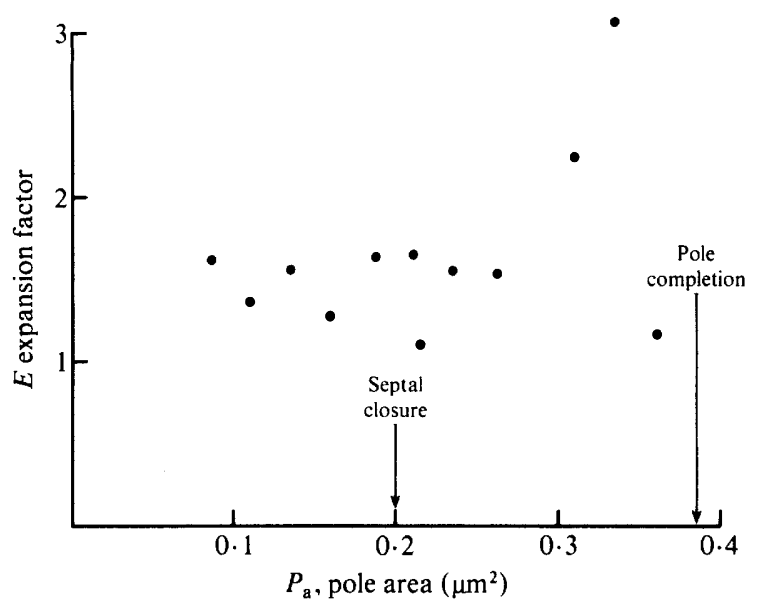

Fig. 1. Expansion factor during pole formation. Data presented by Burdett \& Higgins (1978) were reanalysed as indicated in the text. The abscissa is the area of the developing pole estimated from computer reconstructions of electron micrographs. The arrows show the point of completion of the septum and the pole.

$\pm 0 \cdot 50$. By excluding the last three measurements the statistical estimate becomes $1 \cdot 52 \pm 0 \cdot 17$. Moreover, it can be seen that $E$ has about the same value early in the process, when the septum has not closed, as it has afterwards. Therefore, because $E$ does not change systematically during pole formation, it is concluded that the unsplit septum does not stretch appreciably.

\section{CALCULATED SHAPES OF THE DEVELOPING POLE}

In the first paper of this series (Koch et al., 1981), the shape of the pole of Streptococcus faecium was calculated on the assumption that only the newly split and externalized septal wall is plastic, and that only this region is deformed to accommodate the local physical stress. The model proposed at that time for Streptococcus, which has been further confirmed recently (M. L. Higgins \& A. L. Koch, unpublished), assumed that the externalized wall quickly became rigid and not deformed (toward a spherical shape) during subsequent growth. Our previous studies with B. subtilis (Koch, 1983; Burdett \& Koch, 1984) show that this assumption does not hold for the pole of this species.

\section{If shape changes only during exteriorization}

If the unsplit septum does not stretch at all and if only the newly exteriorized wall is plastic, then the pole as it develops is restricted to a small range of shapes. If an annulus of septum splits, and thus decreases the radius by $d r$, then the area exteriorized is $\pi r^{2}-\pi(r-d r)^{2}$ or $2 \pi r d r$ if second order differentials are neglected. When this material is expanded to achieve an area of $E 2 \pi r d r$, the angle that the newly externalized pole would form with the axis of the cell will be $\sin ^{-1}(1 / E)$. If $E$ were 2, a cone would develop with an angle of $30^{\circ}$. If $E$ were 1.52 , as estimated in the last section for the $B$. subtilis pole, the angle of the cone would be $41.4^{\circ}$. Of course, a conical pole, such as shown in Fig. 2(a), would only form if the previously externalized wall had become rigid instantaneously. Because such cone shapes are only seen in fusiform organisms and never with such a high angle, we assumed in our earlier work (Koch, 1983; Burdett \& Koch, 1984) that actual shapes were non-conical because of stretching of the unsplit septum. The analysis given in the previous section shows that this assumption was wrong. Consequently, we must now assume that unlike the pole wall of Streptococcus the entire pole wall of $B$. subtilis continues to be deformed for some time after it is externalized. 


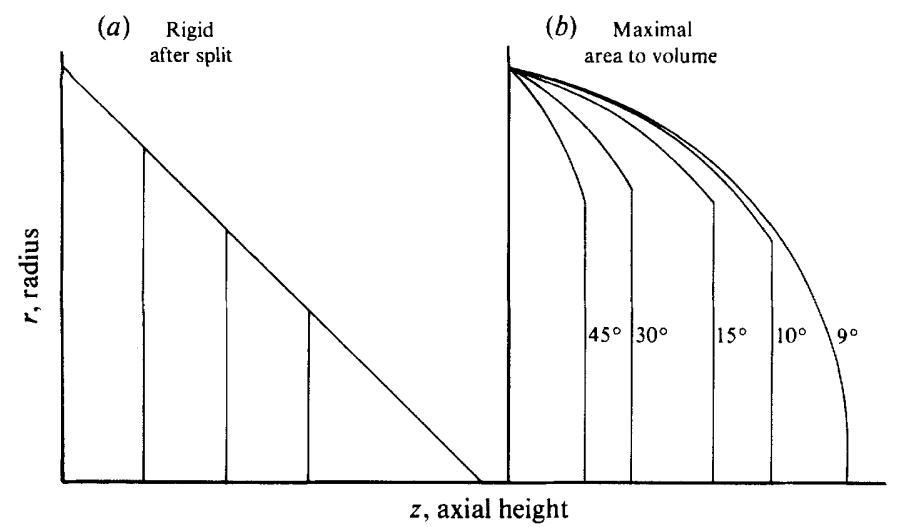

Fig. 2. Hypothetical pole shapes if the cross-wall stretches like a soap bubble film. The figure depicts the profiles which if rotated around the horizontal axis would generate the shape of the pole during various stages of its development. $(a)$ The fusiform shape produced by stretching of the newly exteriorized wall to 1.5 times the area of the unsplit septum. It is also assumed that the unsplit septum does not stretch and then the previously exteriorized polar wall does not further alter its shape after externalization. (b) The polar profile produced if the previously exteriorized wall continues to alter its shape subject to the restriction that the expansion factor is $1 \cdot 5$. Thus, throughout pole formation the area of the exteriorized pole divided by 1.5 plus the septal disc area equals the cross-sectional area of the cylindrical part of the cell. The indicated angles are the initial slope of the pole.

\section{Calculated shape if volume to surface of pole is maximized}

If the wall were stretched as it became externalized and the entire nascent pole were to assume a shape that maximized the nascent pole volume for the available surface area throughout the pole development, then the shape of the externalized wall would be determined by the same factors that would control the shape of a soap bubble. Only after division would the pole shape tend to become spherical. [It would become hemispherical if $E$ were 2, but a spherical segment if $E$ were less than 2.]

Previously (for a review see Koch, 1984) we devised computer programs to calculate the shapes that a bubble with cylindrical symmetry could assume. The problem of predicting the shape of developing poles was solved by computing the pole shapes, various initial tangents to the cylindrical side wall surface were assumed, and projecting the shape of the developing nascent pole. For the present application these projections were halted when the externalized area plus $E \pi D_{\min }{ }^{2} / 4$ equalled the final pole area, $P_{\text {af }}$. Different initial angles corresponded to different stages in pole formation. The results of such computations are shown in Fig. 2(b). The calculated theoretical shape of the nascent poles is a better representation of the developing pole as depicted in Fig. 6 of Burdett \& Higgins (1978). These predicted shapes differ from the observed ones in that they do not have a high enough curvature near the side wall.

\section{Beyond the cone and the sphere}

Because there is more curvature near the edge of the poles, other possibilities need to be considered. One that jumps to mind is that the network structure of the murein fabric of the original cross-wall may constrain the final shape of the pole.

The calculation of the polar shape under these restrictions is a problem in the calculus of variation. It is a difficult problem and probably mathematically intractable. But a good approximation of the shape maximizing the contained volume was obtained by picking a family of curves that all approach the axis perpendicularly and, thus, give a non-pointed pole. The family is generalized by the formula:

$$
\theta=\theta_{0}+\left(90-\theta_{0}\right)\left(1-r^{\mathrm{n}}\right)
$$

where $\theta_{0}$ is the angle at the junction of cylinder and pole, $\theta$ is the angle at that part of the pole where the radius is $r$, and $\mathrm{n}$ is an arbitrary constant. With this formula, the surface area and 


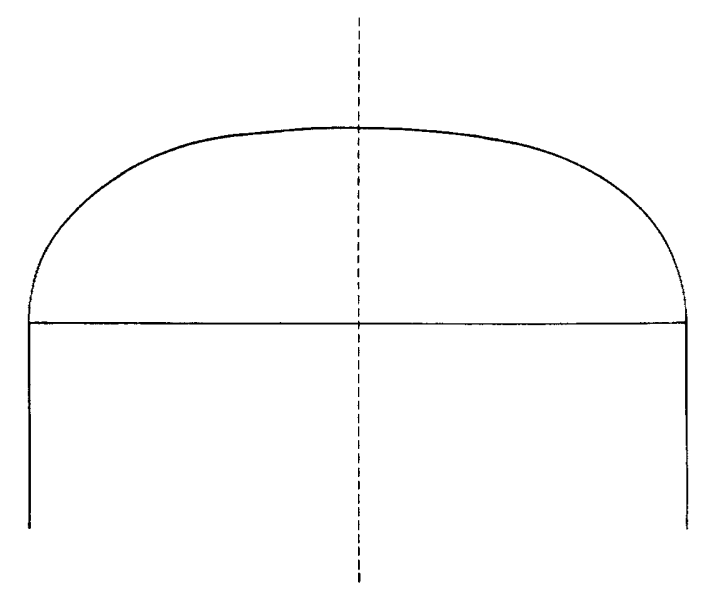

Fig. 3. Hypothetical pole shape if the septum has the stretching qualities of a rubber sheet. Because the cylindrical wall constrains the circumferential expansion of the cross-wall, expansion of area is less for a segment of cross-wall near the perimeter than a segment near the centre (see text). This model fits the experimental data best.

contained volume can be computed and, therefore, the values of $\theta_{0}$ and $\mathrm{n}$ which maximized the volume for any expansion factor can be found by an iterative process. For $E=1.52$ the curve found in Fig. 3 was obtained by a hunt and search procedure.

It can readily be seen why this leads to a polar shape in which most of the curvature is in the region of the cell near the cylindrical surface. The most peripheral septal wall (i.e. the wall which is split first) is constrained in one dimension to have a diameter equal to the diameter of the rod portion of the cell. Therefore, the stresses in the circumferential direction are supported by the rigid cylindrical wall. Consequently, the total increase in area as the wall expands will be made up only by an increase in the axial direction. Thus, the full value of the extensibility of the wall area in this part of the poles cannot be achieved. On the other hand, in the last portion of septum to be split (i.e. the region that will form the tip of the pole) the radial stresses cause expansion equally in all directions. This effect leads to a flat ended pole that meets the side wall at a very small angle, as shown in Fig. 3.

\section{CONCLUSIONS}

The pole of $B$. subtilis has a shape that is consistent with its mode of manufacture: it is stretched from a planar disc. Under the hydrostatic pressure that is developed by the interaction of metabolic processes and wall growth, the cell shape is determined by distribution of wall material. This is analogous to the way that a rubber balloon achieves a special shape when inflated. The Gram-positive rod shape is controlled by its inside-to-outside side wall construction and by the fact that the rod constructs a planar septum. Then, either by the stress pattern that develops therein or by effects produced by the energized cytoplasmic membrane (Kirchner et al., 1984; Koch et al., 1985), the hydrolytic enzymes are directed to bisect the septum, the bonds in the existing murein change their conformation and the bowed-out pole is produced without adding more murein.

Finally, we would like to point out that the 'split and stretch' model is a specific example of the bioengineering principle that is the basis of the surface stress theory. The polymerization of new wall takes place under stress-free conditions and only subsequently supports the stress. In this specific case, the wall expands to achieve the shape of the mature pole.

This work was supported by a Travel Grant from the Burroughs Wellcome Company to A.L.K. Work at Indiana University was supported by NIH under grant GM34222. 


\section{REFERENCES}

ARChibald, A. R. \& CoApes, H. E. (1976). Bacteriophage SP50 as a marker for cell wall growth in Bacillus subtilis. Journal of Bacteriology 125, 11951206.

BurdetT, I. D. J. \& Higgins, M. L. (1978). Studies of pole assembly in Bacillus subtilis by computer reconstruction of septal growth zones seen in central, longitudinal, thin sections of cells. Journal of Bacteriology 133, 959-971.

BurdeTt, I. D. J. \& Koch, A. L. (1984). Shape of nascent and completed poles of Bacillus subtilis. Journal of General Microbiology 130, 1711-1722.

Doyle, R. J., KoCH, A. L. \& CARSTENS, P. H. P. (1983). Cell wall-DNA association in Bacillus subtilis. Journal of Bacteriology 153, 1521-1527.

Higgins, M. L. \& Shockman, G. D. (1976). Studies of a cycle of cell wall assembly in Streptococcus faecalis by three-dimensional reconstructions of thin sections of cells. Journal of Bacteriology 127, 1346-1358.

KIRChNER, G., KoCH, A. L. \& DOYle, R. J. (1984). Energized membrane regulates cell pole formation in Bacillus subtilis. FEMS Microbiology Letters 24, 143147.

$\mathrm{KoCH}$, A. L. (1983). The surface stress theory of microbial shapes. Advances in Microbial Physiology 24, 301-366.
KocH, A. L. (1984). How bacteria get their shapes: the surface stress theory. Comments in Molecular and Cellular Biophysics 2, 179-196.

KoCH, A. L. \& BURdeTt, I. D. J. (1986). Normal pole formation during total inhibition of wall synthesis of Bacillus subtilis. Journal of General Microbiology 132, 3441-3449.

Koch, A. L. \& Doyle, R. J. (1985). Mechanism of inside-to-outside growth and turnover of the wall of the Gram-positive rod. Journal of Theoretical Biology 117, 137-157.

Koch, A. L., Higgins, M. L. \& Doyle, R. J. (1981). Surface tension-like forces determine bacterial shapes: Streptococcus faecium. Journal of General Microbiology 123, 151-161.

KOCH, A. L., KIRChNer, G., DoYle, R. J. \& BURdeTt, I. D. J. (1985). How does a Bacillus split its septum right down the middle? Annales de Microbiologie 136A, 91-98.

Mobley, L. T., Koch, A. L., Doyle, R. J. \& Streips, U. N. (1984). Insertion and fate of cell wall in Bacillus subtilis. Journal of Bacteriology 158, 169-179.

PoOLEY, H. M. (1976). Turnover and spreading of old wall during surface growth of Bacillus subtilis. Journal of Bacteriology 125, 1127-1138. 